\title{
Emergency one-stage resection without mechanical bowel preparation for acute sigmoid volvulus
}

\author{
OO Oludiran ${ }^{a}$ and OC Osime ${ }^{a}$
}

\begin{abstract}
The traditional staged operation for acute sigmoid volvulus is giving way to onestage resection. Similarly, the conventional requirement for bowel cleansing is being increasingly questioned. In this report, we reviewed the outcome of our management over a ten-year period. There were 24 patients aged 23-100 years. Of these, 21 had one-stage primary resection with no clinical anastomotic leak and only one wound infection and fascial dehiscence. The two deaths from this group were due to respiratory failure in a patient aged 100 years and overwhelming sepsis in a younger patient with bowel gangrene from ileosigmoid knotting. We conclude that emergency one-stage resection for acute sigmoid volvulus without mechanical bowel preparation is safe.
\end{abstract}

\section{INTRODUCTION}

Sigmoid volvulus accounts for $8 \%$ of all cases of intestinal obstruction in our environment. ${ }^{1}$ Apart from observed differences in the epidemiological pattern, the clinical presentation is similar to what is seen in other parts of the world. The management of sigmoid volvulus has evolved over the years

KEY WoRDS: Sigmoid volvulus, bowel preparation, primary resection, anastomosis

${ }^{a}$ Department of Surgery, College of Medical Sciences, University of Benin, Benin City, Nigeria.

Correspondence: Oludiran OO, Department of Surgery, College of Medical Sciences, University of Benin, Benin City, Nigeria. E-mail: oludiran@yahoo.com from simple detortion either by endoscopic means or open surgery through colostomyrelated procedure, and now one-stage resectional surgery. Even though endoscopic detortion is a useful initial management in the absence of gangrene or peritonitis, resection of the redundant sigmoid colon remains the definitive treatment.

In 1980, Osime ${ }^{2}$ reviewed 13 cases managed over a five-year period in this centre. Most cases were managed by resection of the redundant sigmoid colon. However colon, the choice of procedure was either a PaulMiculicz exteriorisation resection or a Hartmann's procedure. These required a readmission for a second stage surgery to restore bowel continuity. Although Finsterer ${ }^{3}$ had 
earlier advocated primary resection and anasto-mosis, it did not gain popular support because of the fear of anastomotic failure and wound infection following surgery in the unprepared bowel. However, evidence supporting this fear is lacking. ${ }^{4,5}$ In fact there are studies demonstrating increased risk of wound infection in patients who received bowel preparation. ${ }^{6,7}$ Thus, routine bowel prepara-tion is increasingly been questioned. ${ }^{5,7}$ There is therefore a growing interest in primary resection and anastomosis for obstructive lesions of the colon in the emergency setting.

Few studies in West Africa have been carried out to evaluate the place of this change in our practice. Sule et $\mathrm{al}^{8}$ in 1999 reported a favourable outcome with one-stage resection after on-the-table colonic lavage. However, on-the-table lavage is a time-consuming procedure with the potential of increasing risk from prolonged exposure to anaesthesia. It is also messy, causes some discomfort for the patient and increases the cost of management in the face of scarce resources. At the University of Benin Teaching Hospital, Benin City, Nigeria, we have over the years embarked on a regime of emergency colon resection and primary anastomosis for sigmoid volvulus without any form of bowel preparation. The purpose of this retrospective study is to review the outcome of this practice.

\section{PATIENTS AND METHOD}

The medical records of all patients who were operated on for sigmoid volvulus from January 1992 to December 2001 at the University of Benin Teaching Hospital, Benin City, Nigeria, were reviewed. Their standard demographic data, operative procedure and any complication of treatment were noted.

Diagnosis was made from the medical history and confirmed by emergency plain abdominal x-rays. Ancillary investigations carried out included haematologic profile, serum urea, electrolytes and urinalysis. Chest $\mathrm{x}$-rays and an electrocardiograph were done based on age above 50 years or from observed clinical indication. Initial treatment consisted of nasogastric suction, fluid, electrolyte, acidbase management and urethral catheterisation for hourly urine output monitoring. Pre-operative antibiotics were administered at induction of anaesthesia and continued for three to five days.

At laparotomy, a routine exploration was carried out, diagnosis confirmed and the viability of the bowel was noted. The dilated bowel was decompressed either through a colotomy before resection or through the proximal end of the resected gut. A handsewn two-layered anastomosis was then carried out. The peritoneal cavity was washed out with two litres of warm normal saline. A tube drain was left in the left paracolic gutter beside the anastomosis. At the end of every procedure, we carried out an anal dilatation by the Lord's procedure to reduce intracolonic pressure and relieve tension to the anastomosis.

\section{RESULTS}

Twenty four patients had emergency surgery for acute sigmoid volvulus during the period. This consisted of 20 males and four females. They were aged 23-100 years (mean 51.4) (Table 1). Duration of symptoms before presentation was 1-21 days (mean 5.8). There were two cases of ileosigmoid knotting. A definite pre-operative diagnosis of sigmoid volvulus was made in 13 patients; 10 had only a diagnosis of intestinal obstruction and in one case the clinical diagnosis was peritonitis due to suspected perforated viscus. Six patients had history suggestive of previous episodes. Three patients were known hypertensive and two diabetic. Six patients already developed bowel gangrene at the time of presentation. 
Table 1 Age distribution of the patients

\begin{tabular}{lc}
\hline Age (years) & Number of patients \\
\hline $21-30$ & 6 \\
$31-40$ & 5 \\
$41-50$ & 5 \\
$51-60$ & 2 \\
$61-70$ & 2 \\
$71-80$ & 1 \\
$81-90$ & 2 \\
$91-100$ & 1 \\
\hline Total & 24 \\
\hline
\end{tabular}

Twenty one patients had primary resection and anastomosis without mechanical bowel preparation. One patient had on-the-table lavage and in the remaining two the procedure carried out was resection, anastomosis and protective proximal colostomy. The outcome of surgery is shown in Table 2. There was no clinical anastomotic leak, intraabdominal abscess or recurrence of volvulus. The duration of hospitalisation ranged from 10 to 19 days (mean 12.7) for the patients who had resection and primary anastomosis, whereas those with protective colostomy spent 30-50 days (mean 45) for both the primary surgery and colostomy closure.

There were three deaths (12.5\%) (Table 3). These were in two elderly patients aged 85 and 100 years and another 38-year-old man with an ileosigmoid knotting. The latter had gangrene of $60 \mathrm{~cm}$ of ileum and $30 \mathrm{~cm}$ of sigmoid colon. His pre-operative diagnosis was peritonitis secondary to perforated viscus.

\section{DISCUSSION}

This study has shown that primary resection and anastomosis without faecal diversion in the unprepared large bowel is a safe procedure in the management of sigmoid volvulus without demonstrable increase in risk of anastomotic dehiscence, intra-abdominal abscess or abdominal wound infection. This is similar to the experience of other workers with this management regime for obstructive colonic lesions. ${ }^{9-12}$

\section{Table 2 Outcome of procedure}

\begin{tabular}{lccc}
\hline & Without lavage & $\begin{array}{c}\text { Anastomosis } \\
\text { With lavage }\end{array}$ & With protective colostomy \\
\hline Number of patients & 21 & 1 & 2 \\
Anastomotic leak & - & - & - \\
Wound infection/dehiscence & $1(4.8 \%)$ & - & - \\
Death & $2(9.5 \%)$ & 1 & - \\
\hline
\end{tabular}

Table 3 Analysis of deaths

\begin{tabular}{llll}
\hline Age (years) & Co-morbid problem & ASA classification & Cause of death \\
\hline 100 & Hypertension & IV & Respiratory failure \\
& Proteinuria 2+ & & \\
85 & Hypertension & III & Electrolyte imbalance \\
38 & - & IV & Overwhelming sepsis \\
\hline
\end{tabular}


Routine mechanical preparation of the large bowel remains a standard pre-operative preparation for elective colon surgery. In addition to facilitating anastomosis in a clean field, soilage of anastomosis with faecal matter is reduced. In the emergency situation, routine preparation is impossible. In order to achieve colon cleansing, antegrade intraoperative lavage with electrolyte solution was introduced. Although favoured by many surgeons, its value has been questioned. ${ }^{5,7}$ Apart from causing the patient much discomfort, it adds to the operative risk from prolonged exposure to anaesthesia. The sometimes messy nature can actually cause increased contamination and subsequent wound sepsis. Compared with the experience of Sule et al, ${ }^{8}$ who practiced antegrade lavage in their series (in the middle belt of Nigeria) we experienced less wound infection rate (13.3\% vs. $4.8 \%)$.

We had no case of clinically evident anastomotic dehiscence. This is similar to what was reported by Sule et $\mathrm{al}^{8}$ in their series. In their randomised controlled study, Santos et $\mathrm{al}^{6}$ did not find any significant difference in anastomotic dehiscence rates whether the bowel was prepared or not before elective colon resection. There has also been reports of serious electrolyte disturbance from bowel preparation particularly in elderly patients. ${ }^{13}$ This is similar to our experience with the only patient who had antegrade intraoperative lavage. She was an 85-year-old known hypertensive. She developed persistent hypokalaemia with ileus, symptomatic hypocalcaemia, diarrhoea and eventually died 28 days after surgery.

The operative mortality rate in published works reviewed ranged from 0 to $21.4 \%$. It increases with the age of the patient, the presence of co-morbid diseases and is particularly high for cases of ileosigmoid knotting and when gangrene has ensued. In their study in Guinea, Baragani et al ${ }^{14}$ recorded a mortality of $21.4 \%$ in the presence of gangrene as against 5.8\% without gangrene. Only few of these arise directly as a result of complication related to the colonic surgery. We had an overall mortality of $12.5 \%$. These were from respiratory failure, electrolyte imbalance and overwhelming sepsis. In the absence of gangrene a preliminary endoscopic detortion would possibly have allowed for adequate pre-operative preparation, a planned surgery and possibly better chance of survival than we had for the elderly patients who died in this series.

In conclusion, primary resection and anastomosis of the colon without faecal diversion for acute sigmoid volvulus is safe and can be accomplished without any mechanical bowel preparation. The general condition of very ill patients should be optimised before surgery. The presence of gangrene of the bowel worsens prognosis.

\section{Reference}

1. Osime OC, Okobia MN and Osime U. The changing pattern of intestinal obstruction. Nig J Surg Sci 2002; 12(1): 5-8.

2. U Osime. Volvulus of the sigmoid colon. $J R$ Coll Surg Edinb 1980; 25(1): 32-37.

3. Finsterer H (1912), quoted by U Osime. Volvulus of the sigmoid colon. $J$ R Coll Surg Edinb 1980; 25(1): 32-37.

4. Arspinger RC and Helling TS. An evaluation of colon anastomosis in prepared and unprepared bowel. Clin Gastroenterol 1988; 10(6): 638-641.

5. van Geldere D, Fa-Si-Oen P, Noach LA, Rietra PJ, Peterse JL and Boom RP. Complications after colorectal surgery without mechanical bowel preparation. J Am Coll Surg 2002; 194(1): 40-47.

6. Santos JC, Batista J, Sirimarco MT, Guimaraes AS and Levy CE. Prospective randomised trial of mechanical bowel preparation in patients undergoing elective colorectal surgery. Br J Surg 1994; 81(11): 1673-1676. 
7. Platell C and Hall J. What is the role of mechanical bowel preparation in patients undergoing colorectal surgery? Dis Colon Rectum 1998; 41(7): 875-882.

8. Sule AZ, Iya P, Obekpa PO, Ogbonna B, Momoh JT and Ugwu BT. One stage procedure in the management of acute sigmoid volvulus. J R Coll Surg Edinb 1999; 44(6): 164-166.

9. Hsu TC. One stage resection and anastomosis for acute obstruction of the left colon. Dis Colon Rectum 1998; 41(1): 28-32.

10. Kuzu MA, Aslar AK, Soran A, Polat A, Topcu $\mathrm{O}$ and Hengimen S. Emergent resection for acute sigmoid volvulus: results of 106 consecutive cases. Dis Colon Rectum 2002; 45(8): 1085-1090.
11. Duthie GS, Forster ME, Price-Thomas JM and Leaper DJ. Bowel preparation or not for elective colorectal surgery. J R Coll Surg 1990; 35(3): 169-171.

12. Naraysingh V, Rampaul Maharaj D, Kuruvilla T, Ramcharan K and Pouchet B. Prospective randomized trial of primary anastomosis without colonic lavage for patients with obstructed left colon. Br J Surg 2000; 87(3): 376-377.

13. Boivin MA and Kahn SR. Symptomatic hypocalcaemia from oral sodium phosphate: a report of two cases. Am J Gastroenterol 1998; 93(12): 2577-2579.

14. Baragani M, Conde AS, Longo R, Italiano A, Terenzi A and Venuto G. Sigmoid volvulus in West Africa: a prospective randomized study on Surgical treatment. Dis Colon Rectum 1993; 36(2): 186-190. 